\title{
Modeling Alveolar Volume Changes During Periodic Breathing in Heterogeneously Ventilated Lungs
}

\author{
Sara-Jane Dunn and Jonathan P. Whiteley \\ Oxford University Computing Laboratory, Wolfson Building, Parks Road, Oxford OX1 3QD, UK
}

(Received 3 February 2010; accepted 30 March 2010; published online 13 April 2010)

Associate Editor John H. Linehan oversaw the review of this article.

\begin{abstract}
A simplified model of periodic breathing, proposed by Whiteley et al. (Math. Med. Biol. 20:205-224, 2003), describes a non-uniform breathing pattern for a lung with an inhomogeneous gas distribution, such as that observed in some subjects suffering from respiratory disease. This model assumes a constant alveolar volume, and predicts incidence of irregular breathing caused by small, poorly ventilated regions of the lung. Presented here is an extension to this work which, by allowing variable lung volume, facilitates the investigation of pulmonary collapse in poorly ventilated compartments. A weakness of the original model is that a very small alveolar volume is required for periodic breathing to occur. The model presented within, which removes the assumption of constant compartment volume and allows alveolar volume to vary with time, predicts periodic breathing at higher, more realistic alveolar volumes. Furthermore, the predicted oscillations in ventilation match experimental data more closely. Thus the model that allows for alveolar collapse has improved upon these earlier results, and establishes a theoretical link between periodic breathing and atelectasis.
\end{abstract}

Keywords-Delay differential equations, Atelectasis.

\section{INTRODUCTION}

An irregular breathing pattern is observed in many subjects with respiratory disease, characterized by periodic oscillations in ventilation, which in severe cases can be punctuated by periods of apnoea-a condition known as Cheyne-Stokes breathing. ${ }^{14}$ Periodic breathing, while observed in healthy subjects during sleep, acclimatization to high altitudes, and in those under anesthesia, is also associated with a number of pathological problems, such as heart failure, stroke, and neurological disease. ${ }^{22}$

Address correspondence to Sara-Jane Dunn, Oxford University Computing Laboratory, Wolfson Building, Parks Road, Oxford OX13QD, UK. Electronic mail: sara-jane.dunn@comlab.ox.ac.uk, jonathan.whiteley@comlab.ox.ac.uk
A number of mathematical models have been developed to study periodic breathing, ${ }^{1,4,7,8,18,24}$ many of which derive from the general Grodins model for cardiorespiratory control, ${ }^{10}$ which is a compartment model based on the hypothesis that the periodicities associated with ventilation are the result of feedback delays. These delays arise due to the physical distance that the blood has to travel from the lungs to the chemoreceptors which act to coordinate ventilatory response, causing them to react to past conditions. Such existing models, while generating periodicities in ventilation, do not capture the Cheyne-Stokes oscillations accurately. Model results show regular oscillations, whereas the clinically observed pattern usually displays periods of slight breathing, punctuated by short bursts of deep breathing with period of approximately $40-60 \mathrm{~s}^{14}$

Many previous models of periodic breathing are based on simplifications of the Grodins model that consider only carbon dioxide partial pressures. However, periodicities in arterial blood oxygen concentration have been observed in subjects exhibiting periodic breathing, which are uniformly associated with low arterial oxygen partial pressure. ${ }^{9,19,20}$ Whiteley et al. ${ }^{24}$ investigate the chemosensory response to oxygen signals and the subsequent effect on ventilation under such conditions. Mismatch between inspired ventilation and perfusion inside the lungs, often the consequence of respiratory illness, leads to inhomogeneity in the transfer of gas and low arterial blood oxygen partial pressure. To account for this, the Grodins model is adjusted to consider a lung with two compartments - one healthy region, and a second, poorly ventilated region. The results identify scenarios under which periodic breathing is likely to occur: namely small, poorly ventilated lung compartments. Further to this, the results indicate that the volume of the poorly ventilated compartment must be sufficiently small to allow the onset of periodic breathing. By 
definition, a large, poorly ventilated alveolar compartment will contain a large volume of gas with concentration that is not affected much due to ventilation, and so variations in gas concentrations leaving the lung will be smoothed out. This compartment will therefore exhibit a smaller rate of change of oxygen concentration in response to ventilation than a smaller compartment, and thus the effect on the overall partial pressure of oxygen leaving the lung will not be significant. Subsequently, the blood arterial oxygen concentration change between the chemoreceptors and blood leaving the lung will be small, and periodic breathing will not occur. If rather, such a large compartment were to be better ventilated, then the alveolar partial pressure of oxygen would be higher, leading to a higher arterial oxygen partial pressure. Under these conditions, blood leaving the compartment would lie on the flat portion of the oxygen dissociation curve and again, periodic breathing will not occur.

Poorly ventilated regions of the lung, such as those described by the model presented by Whiteley et al., ${ }^{24}$ are vulnerable to collapse, a condition known as atelectasis. ${ }^{3}$ This occurs when the net flux of gas across the alveolar membrane into the blood is greater than that supplied by inspired ventilation, causing the lung volume to decrease. Models exist that examine the onset and aggravation of atelectasis ${ }^{11,12}$ and such work has lead to the conclusion that increasing the fraction of inspired oxygen reduces the time to collapse.

The model presented within this article is an extension of the simplified Grodins model proposed by Whiteley et al. ${ }^{24}$ (henceforth referred to as the Reduced Model) to allow for variable alveolar compartment volume. The model thus facilitates investigation of the collapse of poorly ventilated regions of the lung, as seen in subjects suffering from respiratory disease. In contrast to existing models of atelectasis, pulmonary collapse is considered in the presence of impaired, rather than eliminated, ventilation and under conditions of low arterial oxygen partial pressure. Firstly, the Reduced Model is presented and the results discussed. Subsequently, the equations are extended to form a model that takes into account the change in volume of a poorly ventilated lung compartment over time, and hence permits alveolar collapse. Simulations using this model, together with a response analysis for critical system parameters, are evaluated and discussed.

\section{THE MODEL OF PERIODIC BREATHING}

The periodic breathing model proposed by Whiteley et al. $^{24}$ is based on the Grodins model of cardio-respiratory control, a general compartmental model describing the oxygen, nitrogen, and carbon dioxide
TABLE 1. The variables used and their units.

\begin{tabular}{lll}
\hline Variable & \multicolumn{1}{c}{ Description } & \multicolumn{1}{c}{ Units } \\
\hline$F$ & Fractional concentration of $\mathrm{O}_{2}$ & Dimensionless \\
$G$ & Fractional concentration of $\mathrm{CO}_{2}$ & Dimensionless \\
$P$ & Partial pressure & $\mathrm{mmHg}^{-1}$ \\
$Q$ & Perfusion & $\mathrm{L} \mathrm{min}^{-1}$ \\
$\dot{V}$ & Ventilation & $\mathrm{L} \mathrm{min}^{-1}$ \\
$S$ & Saturation & Dimensionless \\
$C$ & Blood gas content & Liter gas per liter blood \\
\hline
\end{tabular}

TABLE 2. Subscripts used in this study.

\begin{tabular}{ll}
\hline Subscript & \multicolumn{1}{c}{ Description } \\
\hline $\mathrm{A}$ & Alveolar \\
$\mathrm{B}$ & Brain \\
$\mathrm{O}_{2}$ & Oxygen \\
$\mathrm{CO}_{2}$ & Carbon dioxide \\
$\mathrm{a}$ & Arterial blood \\
$\bar{v}$ & Mixed venous blood \\
$\mathrm{I}$ & Inspired \\
$\mathrm{E}$ & Expired \\
1 & Poorly ventilated lung compartment \\
2 & Healthy lung compartment \\
\hline
\end{tabular}

concentrations in the lungs, brain, tissues, and cerebrospinal fluid. ${ }^{10}$ Grodins et al. assume that the lung may be modeled by a constant alveolar volume containing well-mixed gases with homogeneous concentrations. Whiteley et al. investigate periodicities in ventilation caused by low levels of arterial oxygen partial pressure by constructing a simplified version of the Grodins model that takes into account inhomogeneities in ventilation, perfusion, and gas exchange for oxygen only. This model is introduced in the section "The Reduced Model," given that it forms the basis for the model of periodic breathing that allows for alveolar collapse, which follows in the section "Incorporating variable compartment volume into the model." For reference, the relevant variables and the subscripts that refer to the appropriate gas and compartment for both models are outlined in Tables 1 and 2, respectively.

\section{The Reduced Model}

Those suffering from respiratory conditions often exhibit an inhomogeneous distribution of gas concentrations in the lung, caused by an inhomogeneous distribution of ventilation and perfusion. ${ }^{24}$ To allow for this, the lung is defined to have two homogeneous compartments, one healthy region and a poorly ventilated region, which each receive a constant proportion of the total inspired ventilation. Nitrogen is a passive variable that decouples from the Grodins model $^{6}$; as such it is independent of the remaining variables, and so is not required for their determination. 
In addition to this, under low arterial oxygen partial pressure conditions, the equations for oxygen also decouple. $^{8}$ Due to the normal ventilation-perfusion ratio in the healthy compartment, blood leaving this compartment will be almost fully oxygen saturated and will therefore have content that lies on the flat portion of the oxygen dissociation curve. ${ }^{24}$ A change in ventilation or the fraction of inspired oxygen will have a negligible effect on the oxygen content and thus the content leaving the healthy compartment is assumed constant and equal to that of fully saturated blood. Consequently, the total arterial oxygen content, $C_{\mathrm{a}}$, is only dependent on the oxygen content in the blood leaving the poorly ventilated compartment, and the proportion of blood that flows to each alveolar unit.

The Reduced Model thus considers only one equation, a delay differential equation modeling the change in concentration of oxygen in the poorly ventilated compartment, which is denoted by the subscript ' 1 ':

$$
\begin{aligned}
V_{\mathrm{A}_{1}} \frac{d F_{\mathrm{A}_{1}}}{d t}= & v_{1} \dot{V}\left(F_{\mathrm{A}_{1}}(t-\tau)\right)\left(F_{I}-F_{\mathrm{A}_{1}}(t)\right) \\
& +K Q_{1}\left(C_{\bar{v}}-C_{\mathrm{A}_{1}}(t)\right),
\end{aligned}
$$

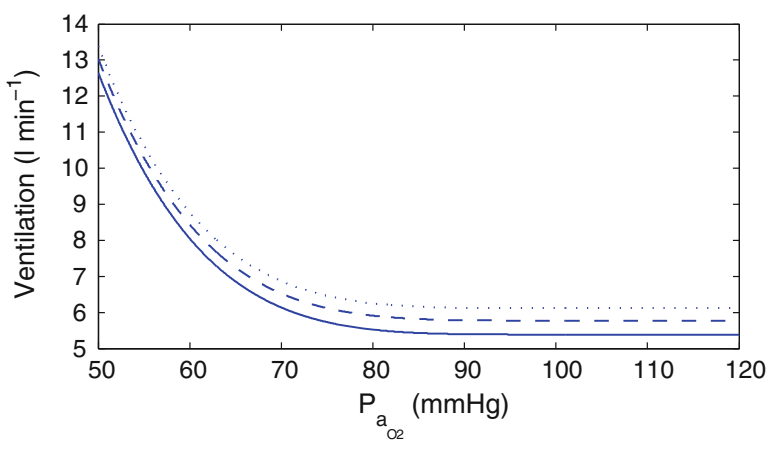

FIGURE 1. Plot of the ventilation function defined by Grodins et al. ${ }^{10}$ for $P_{\mathrm{BCO}_{2}}=43 \mathrm{mmHg}$ (solid line), $44 \mathrm{mmHg}$ (dashed line), $45 \mathrm{mmHg}$ (dotted line). This plot demonstrates that the function has a greater dependence on $P_{\mathrm{aO}_{2}}$ than on $P_{\mathrm{BCO}_{2}}$ for low $P_{\mathrm{aO}_{2}}$.

dependent on the arterial oxygen partial pressure at an earlier time determined by the delay, $\tau$, defined above. $\dot{V}_{0}$ is the gas flow rate for a healthy subject, which takes a constant value. Above $104 \mathrm{mmHg}$, oxygen levels are defined as healthy, ${ }^{10}$ and this function ensures that ventilation increases only in response to a low blood oxygen level by the definition

$$
\left[104-P_{\mathrm{a}}(t-\tau)\right]_{+}^{4.9}= \begin{cases}104-P_{\mathrm{a}}(t-\tau), & \text { if } P_{\mathrm{a}}(t-\tau)<104 \mathrm{mmHg}, \\ 0, & \text { if } P_{\mathrm{a}}(t-\tau)>104 \mathrm{mmHg}\end{cases}
$$

where $V_{\mathrm{A}_{1}}$ is the volume of the compartment, $F_{\mathrm{A}_{1}}$ the fractional concentration of oxygen, $v_{1}$ the proportion of the total inspired oxygen supplied to this compartment, $\dot{V}$ the total inspired ventilation, which is a function of $F_{\mathrm{A}_{1}}(t-\tau), \tau$ the delay corresponding to the time taken for the blood to reach the peripheral chemoreceptors, $F_{\mathrm{I}}$ the fraction of inspired oxygen, $K$ the conversion factor required to change the units from standard temperature and pressure to body temperature and pressure, $Q_{1}$ the perfusion, $C_{\bar{v}}$ the oxygen content in the mixed venous blood, and $C_{\mathrm{A}_{1}}$ the oxygen content in the poorly ventilated compartment.

The fractional concentration of oxygen in the poorly ventilated compartment is related to the arterial partial pressure, $P_{\mathrm{a}}$, via auxiliary equations that are defined in Appendix A. Consequently, the functional form of the ventilation follows that proposed by Grodins et al., where it is defined as a function of $P_{\mathrm{a}}$, and which is plotted in Fig. 1. This function is more dependent on $P_{\mathrm{aO}_{2}}$ than $P_{\mathrm{BCO}_{2}}$ at low $P_{\mathrm{aO}_{2}}$, and thus is adjusted to be solely dependent on blood oxygen levels.

$$
\dot{V}=\dot{V}_{0}+23.6 \times 10^{-9}\left[104-P_{\mathrm{a}}(t-\tau)\right]_{+}^{4.9},
$$

where the units of $\dot{V}$ are $\mathrm{L} \mathrm{min}^{-1}$, and the units of partial pressure are $\mathrm{mmHg}$. Here, ventilation is
For a suitable choice of parameters, the Reduced Model exhibits regular oscillations in $F_{\mathrm{A}_{1}}, P_{\mathrm{aO}_{2}}$ and $\dot{V}$, which are in agreement with some experimental data. ${ }^{9,19,20}$ However, one weakness of this work is revealed in the shape of the oscillations of the ventilation function, which are regular and indicate that a subject alternates between light and deep breathing with equal duration. As outlined earlier, this is not the case for a subject suffering from periodic breathing, which is characterized by short bursts of high ventilation interspersed with longer periods of low ventilation.

Past studies have concluded that lung volume has a great influence on the stability of the respiratory system, with the system being regarded as unstable at the onset of periodic breathing that can continue indefinitely. ${ }^{16,22}$ Mathematically, this corresponds to an unstable steady-state solution of the differential equation, which manifests as periodic oscillations in ventilation. In agreement with this conclusion, the Reduced Model results indicate that the volume of the poorly ventilated compartment is critical in determining the onset of periodic breathing. Indeed, the solutions for $F_{\mathrm{A}_{1}}, P_{\mathrm{aO}_{2}}$ and $\dot{V}$ are more oscillatory for smaller volumes, and crucially, there is a volume above which periodic oscillations cease. Specifically, for the choice of parameters used by Whiteley et al., ${ }^{24}$ it is required 
that $V_{\mathrm{A}_{1}}$ be less than about $0.17 \mathrm{~L}$ for periodic breathing to occur. For a total lung gas volume of $2.5 \mathrm{~L}$, this represents a volume fraction of $6.8 \%$ which is perfused by $50 \%$ of the total blood flow, and reveals a second weakness of the model: periodic solutions only exist for a compartment volume that is rarely seen and is very restrictive, and hence too small to be commonly physiologically plausible.

\section{Incorporating Variable Compartment Volume into the Model}

A key assumption of the Reduced Model is constant alveolar volume, by which inspired and expired ventilation are necessarily equal. Poorly ventilated lung units - such as those that are the cause of periodic breathing - are likely to collapse due to atelectasis, especially for high oxygen concentrations. ${ }^{11,12}$ By allowing compartment volume to vary, and therefore to be dependent on the ventilation of the lung, the effect that lung volume has on periodic breathing can be investigated. Subsequently, whether there is a link to the prevention, or delay, of total lung collapse may be determined.

\section{The Governing Delay Differential Equations}

To incorporate atelectasis into the Reduced Model, the assumption of equal inspired and expired ventilation is relaxed. Consequently, the model is extended to consider the effect of carbon dioxide, thus permitting the calculation of the net flux of gas across the alveolar membrane into the blood. Given that nitrogen is insoluble, and thus decouples from the original Grodins model, it shall not be considered here. $F_{\mathrm{A}_{1}}$ continues to represent the fractional concentration of oxygen in the poorly ventilated region, and $G_{\mathrm{A}_{1}}$ is defined to be the fractional concentration of carbon dioxide. There are now two conservation of mass equations to consider, which describe the rate of change of volume of both oxygen and carbon dioxide inside each compartment:

$$
\begin{gathered}
\frac{d}{d t}\left(V_{\mathrm{A}_{1}} F_{\mathrm{A}_{1}}\right)=\dot{V}_{\mathrm{I}_{1}} F_{\mathrm{I}}-\dot{V}_{\mathrm{E}_{1}(+)} F_{\mathrm{A}_{1}}+K Q_{1}\left(C_{\bar{v}}-C_{\mathrm{A}_{1}}\right) \\
\frac{d}{d t}\left(V_{\mathrm{A}_{1}} G_{\mathrm{A}_{1}}\right)=-\dot{V}_{\mathrm{E}_{1}(+)} G_{\mathrm{A}_{1}}+K Q_{1}\left(D_{\bar{v}}-D_{\mathrm{A}_{1}}\right)
\end{gathered}
$$

These equations have been adjusted to account for non-constant alveolar volume, such that the $V_{\mathrm{A}_{1}}$ term remains inside the time derivative on the LHS. It is assumed that there is zero carbon dioxide in inspired air and now $\dot{V}_{\mathrm{I}_{1}}$ and $\dot{V}_{\mathrm{E}_{1}(+)}$ denote the inspired and expired ventilations for the poorly ventilated compartment, respectively, which are not assumed equal. $C_{\bar{v}}, D_{\bar{v}}$ are the oxygen and carbon dioxide content in the mixed venous blood being returned to the compartment, and $C_{\mathrm{A}_{1}}, D_{\mathrm{A}_{1}}$ the oxygen and carbon dioxide content in the blood leaving the compartment by perfusion. The quantity $\dot{V}_{\mathrm{E}_{1}(+)}$ that appears in Eqs. (3) and (4) is now described. A positive expired ventilation implies that gas is flowing out of the compartment, but should the net flow of gas to the poorly ventilated compartment be negative, then the calculated expired ventilation will subsequently be negative, as explained in the section "Alveolar volume." In this instance, this quantity is set to zero in Eqs. (3) and (4), and it is assumed that it causes a decrease in the volume of the poorly ventilated compartment. Hence, the following notation is adopted.

$$
\dot{V}_{\mathrm{E}_{1}(+)}= \begin{cases}\dot{V}_{\mathrm{E}_{1}}, & \text { if } \dot{V}_{\mathrm{E}_{1}}>0, \\ 0, & \text { otherwise. }\end{cases}
$$

How $\dot{V}_{\mathrm{E}_{1}}$ is calculated and its effect on $V_{\mathrm{A}_{1}}$ is now discussed.

\section{Ventilation}

The total inspired ventilation, $\dot{V}_{\mathrm{I}}$, is again defined using Eq. (2). However, an alveolar deadspace exists that arises due to causes distal to the alveolar-bronchiolar junction, which must be taken into consideration when determining $\dot{V}_{\mathrm{I}_{1}}$. 5 ,21 This comprises the effects due to both the mismatch of ventilation and perfusion within the poorly ventilated compartment, and uneven distribution of ventilation and perfusion between the healthy and unhealthy compartments. This is captured by the inclusion of a constant correction term, $\delta_{\mathrm{s}}$, that models the volume of gas that remains in the deadspace (see Fig. 2). Hence, the amount of oxygen entering the poorly ventilated compartment is less than the fraction of oxygen in the total inspired ventilation, and is given by

$$
\dot{V}_{\mathrm{I}_{1}}=v_{1} \dot{V}_{I}\left(F_{\mathrm{A}_{1}}(t-\tau)\right)-\delta_{\mathrm{s}} .
$$

$\delta_{\mathrm{s}}$ is a model parameter to be determined, the value of which is discussed in the section "Effect of $\delta_{\mathrm{s}}$." Equating the net flux of gas into the poorly ventilated unit,

$$
\dot{V}_{\mathrm{I}_{1}}+K Q_{1}\left(C_{\bar{v}}+D_{\bar{v}}\right)=\dot{V}_{\mathrm{E}_{1}}+K Q_{1}\left(C_{\mathrm{A}_{1}}+D_{\mathrm{A}_{1}}\right),
$$

which is used to find the expired ventilation of the poorly ventilated compartment. 


\section{Alveolar Volume}

Pulmonary collapse occurs due to a reduction in alveolar volume when the net flux of gas into the lung is negative. Utilizing Eq. (6), the change in volume of the poorly ventilated compartment is modeled as dependent on this flux balance, and is defined to have initial volume $V_{\mathrm{A}_{1} \text { rest }}$, which this lung compartment seeks to maintain. The expired ventilation quantifies the balance between the amount of inspired gas and gas returned to the compartment by the mixed-venous blood, with that diffusing out of the compartment. So for the expired ventilation to be positive, the net flow of gas to the compartment must be positive, and the compartment volume will remain constant and equal to $V_{\mathrm{A}_{1} \text { rest }}$ due to the action of breathing out. Should expired ventilation be negative, the reverse holds and the compartment volume will decrease. The rate of decrease is modeled as being directly proportional to this negative flux, so that the volume decreases according to the imbalance. If, however, the expired ventilation becomes positive again, but the volume of the compartment is still lower than its resting value, then it shall increase at a rate proportional to this positive flux. This is captured by

$$
\frac{d V_{\mathrm{A}_{1}}}{d t}= \begin{cases}0 & \text { if } \dot{V}_{\mathrm{E}_{1}}>0 \text { and } V_{\mathrm{A}_{1}}=V_{\mathrm{A}_{1} \text { rest }}, \\
\dot{V}_{\mathrm{E}_{1}} & \text { if }\left\{\begin{array}{l}
\dot{V}_{\mathrm{E}_{1}}<0 \text { or } \\
\dot{V}_{\mathrm{E}_{1}}>0 \text { and } V_{\mathrm{A}_{1}}<V_{\mathrm{A}_{1} \text { rest }} .
\end{array}\right.\end{cases}
$$

Given that the extension of the previous model outlined here now allows the volume of the poorly ventilated compartment to decrease, periodic breathing is more likely to occur.

Equations (3), (4), and (7) complete the mathematical representation of the model of periodic breathing that allows for alveolar collapse.

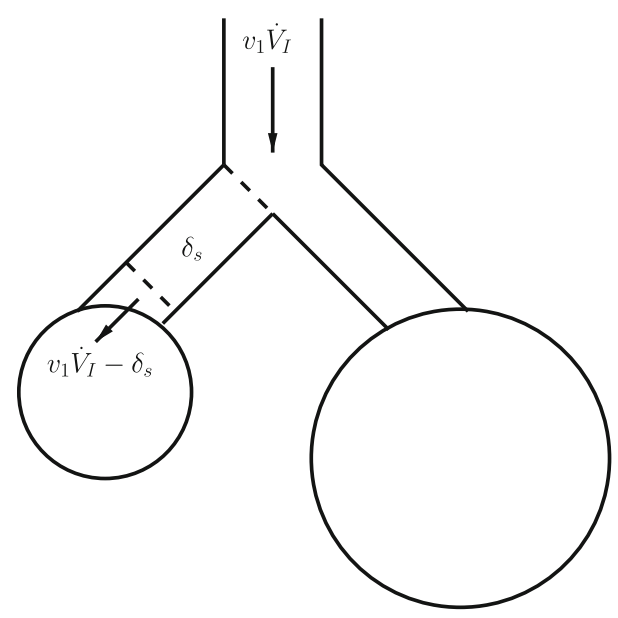

FIGURE 2. An illustration of the proportion of inspired ventilation that remains in the alveolar deadspace associated with the poorly ventilated compartment, which is indicated by the smaller of the two compartments.

\section{RESULTS}

\section{Summary of Computations}

The method for solving Eqs. (3), (4), and (7) is outlined in Appendix A. Unless stated otherwise, in all simulations in this study all parameters take the values given in Table 3. At the chosen resting volume, $V_{\mathrm{A}_{1} \text { rest }}=0.3 \mathrm{~L}$, periodic breathing does not occur for the Reduced Model, and so oscillations in $F_{\mathrm{A}_{1}}$ that may arise do so as a consequence of the extensions to the model. The percentage of inspired oxygen is increased to $F_{\mathrm{I}}=0.8$, in agreement with experimental observation that the incidence of pulmonary collapse is greater with higher levels of inspired oxygen, even in the absence of restrictions in ventilation. ${ }^{11,12}$ Indeed, it has been observed that atelectasis can result from high levels of inspired oxygen. ${ }^{13}$ It should be noted that the parameters used to produce these solutions do not lead toward total lung collapse.

\section{The New Model}

The solutions of the model of periodic breathing that allows for alveolar collapse are shown in Fig. 3.

TABLE 3. The parameter values required for both the Reduced Model and the Extended Model.

\begin{tabular}{|c|c|c|}
\hline Parameter & Value & References \\
\hline$P_{\mathrm{B}}$ & $760 \mathrm{mmHg}$ & Grodins et al. ${ }^{10}$ \\
\hline$P_{\mathrm{H}_{2} \mathrm{O}}$ & $47 \mathrm{mmHg}$ & Carley and Shannon ${ }^{2}$ \\
\hline$P_{\mathrm{A}_{2}}$ & $100 \mathrm{mmHg}$ & Whiteley et al. ${ }^{23}$ \\
\hline$K_{\mathrm{Hb}}$ & 0.2 & Grodins et al. ${ }^{10}$ \\
\hline$\alpha$ & $2.3 \times 10^{-5} \mathrm{~cm}^{2} \mathrm{~s}^{-1}$ & Whiteley et al. ${ }^{23}$ \\
\hline$a_{1}$ & $-8.5322289 \times 10^{3}(\mathrm{mmHg})^{3}$ & Whiteley et al. ${ }^{23}$ \\
\hline$a_{2}$ & $2.1214010 \times 10^{3}(\mathrm{mmHg})^{2}$ & Whiteley et al. ${ }^{23}$ \\
\hline$a_{3}$ & $-6.7073989 \times 10 \mathrm{mmHg}$ & Whiteley et al. ${ }^{23}$ \\
\hline$a_{4}$ & $9.3596087 \times 10^{5}(\mathrm{mmHg})^{4}$ & Whiteley et al. ${ }^{23}$ \\
\hline$a_{5}$ & $-3.1346258 \times 10^{4}(\mathrm{mmHg})^{3}$ & Whiteley et al. ${ }^{23}$ \\
\hline$a_{6}$ & $2.3961674 \times 10^{3}(\mathrm{mmHg})^{2}$ & Whiteley et al. ${ }^{23}$ \\
\hline$a_{7}$ & $-6.7104406 \times 10 \mathrm{mmHg}$ & Whiteley et al. ${ }^{23}$ \\
\hline$Q_{1}$ & $2 \mathrm{~L} \min ^{-1}$ & Whiteley et al. ${ }^{24}$ \\
\hline$Q_{2}$ & $2 \mathrm{Lmin}^{-1}$ & Whiteley et al. ${ }^{24}$ \\
\hline$\dot{V}_{0}$ & $4 \mathrm{Lmin}^{-1}$ & Whiteley et al. ${ }^{24}$ \\
\hline$K$ & 1.21 & Lumb $^{17}$ \\
\hline$v_{1}$ & 0.12 & Whiteley et al. ${ }^{24}$ \\
\hline$Q_{0}$ & $4 \mathrm{~L} \min ^{-1}$ & Whiteley et al. ${ }^{24}$ \\
\hline$C_{\bar{v}}$ & $0.11 \mathrm{~mL} \mathrm{O}_{2} \mathrm{~mL}^{-1}$ blood & Whiteley et al. ${ }^{24}$ \\
\hline$\tau$ & $16.89 \mathrm{~s}$ & Whiteley et al. ${ }^{24}$ \\
\hline$V_{\mathrm{A}_{1} \text { rest }}$ & $0.3 \mathrm{~L}$ & \\
\hline$F_{1}$ & 0.8 & Joyce et al. ${ }^{11,12}$ \\
\hline$P_{\bar{v} \mathrm{CO}_{2}}$ & $46 \mathrm{mmHg}$ & Lumb $^{17}$ \\
\hline$\lambda$ & $\begin{array}{l}3.72 \mathrm{~mL} \mathrm{CO}_{2} \text { per } \mathrm{mL} \\
\text { blood per atm }\end{array}$ & Whiteley et al. ${ }^{25}$ \\
\hline$G_{0}$ & 0.07 & Whiteley et al. ${ }^{23}$ \\
\hline$\delta_{\mathrm{s}}$ & $0.055 \mathrm{~L}$ & \\
\hline
\end{tabular}

These parameters are used in all simulations unless otherwise stated. 

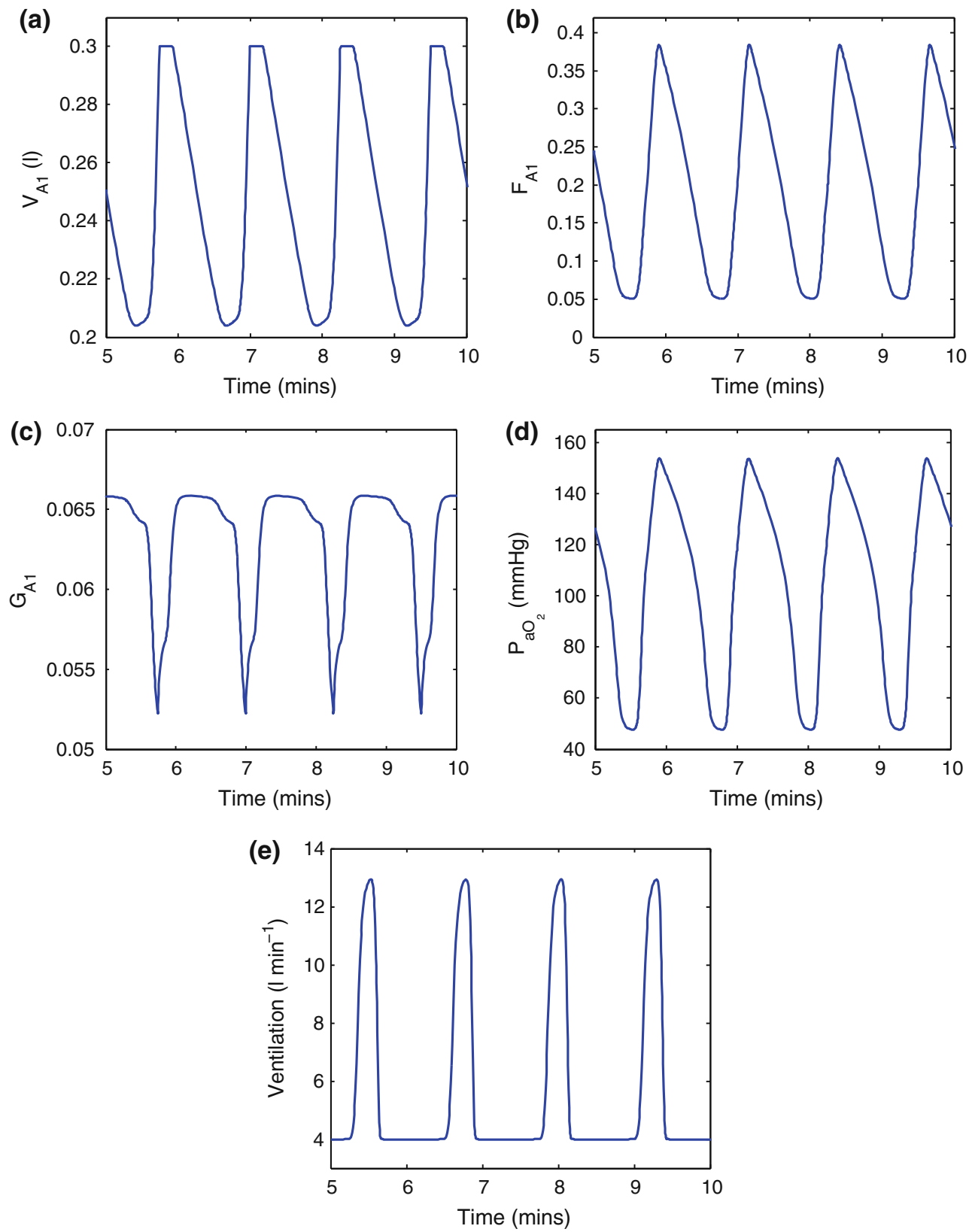

FIGURE 3. Simulations of the model for periodic breathing that allows for alveolar collapse. (a) Oscillations in the volume of the poorly ventilated compartment, $V_{\mathrm{A}_{1}}$; (b) oscillations in the fractional concentration of oxygen, $F_{\mathrm{A}_{1}} ;$ (c) oscillations in the fractional concentration of carbon dioxide, $G_{\mathrm{A}_{1}}$; (d) oscillations in the arterial oxygen partial pressure, $P_{\mathrm{aO}_{2}} ;(\mathrm{e})$ oscillations in ventilation, $\boldsymbol{V}_{1}$.

Oscillations are found in each of the three variables, $V_{\mathrm{A}_{1}}, F_{\mathrm{A}_{1}}$ and $G_{\mathrm{A}_{1}}$, as well as in $P_{\mathrm{aO}_{2}}$ and $\dot{V}_{\mathrm{I}}$, which are dependent on $F_{\mathrm{A}_{1}}$. The initial transient behavior is omitted to show the stable oscillatory cycle exhibited by each variable after a short time, and so the results are presented once $5 \mathrm{~min}$ has elapsed. Overall, these results show that periodic oscillations in lung volume and the fractional concentration of carbon dioxide accompany those in $F_{\mathrm{A}_{1}}, P_{\mathrm{aO}_{2}}$ and $\dot{V}_{\mathrm{I}}$, with a period of approximately $60 \mathrm{~s}$. The oscillations in $F_{\mathrm{A}_{1}}, P_{\mathrm{aO}_{2}}$ and $V_{\mathrm{A}_{1}}$ are synchronized - from the peak values, the variables start to decrease together, but the $V_{\mathrm{A}_{1}}$ oscillations are limited by $V_{\mathrm{A}_{1} \text { rest }}$ and so have a flat peak. The peaks in ventilation occur when $F_{\mathrm{A}_{1}}, P_{\mathrm{aO}_{2}}$ and $V_{\mathrm{A}_{1}}$ are minimum, and the periods of low ventilation occur when these variables are greater than their respective minima. It can be noted that the increase in ventilation precedes the minima of the remaining variables, and that it attains its minimum just before $V_{\mathrm{A}_{1}}$ reaches saturation and therefore before $F_{\mathrm{A}_{1}}$ and $P_{\mathrm{aO}_{2}}$ are maximum. 
In these results $V_{\mathrm{A}_{1} \text { rest }}=0.3 \mathrm{~L}$, and immediately it is clear that this model allows periodic breathing at a higher compartment volume than the Reduced Model. An important result to note is the shape of the oscillation for the total inspired ventilation, $\dot{V}_{\mathrm{I}}$, shown in Fig. 3e. Periods of constant low ventilation, the resting ventilation $\dot{V}_{0}$, are punctuated by bursts of high ventilation, characteristic of the nature of Cheyne-Stokes breathing. A response analysis of the model to critical system parameters is now presented, and in each case the results are presented after $25 \mathrm{~min}$ has elapsed within the simulation, in order to demonstrate the stable oscillatory behavior.

\section{Comparison of the New Model with the Reduced Model}

Figures $4 \mathrm{a}-4 \mathrm{~d}$ compare the oscillations observed in the inspired ventilation function, $\dot{V}_{\mathrm{I}}$, for the Reduced Model and the new model, for increasing compartment volumes $V_{\mathrm{A}_{1}}=V_{\mathrm{A}_{1} \text { rest }}=0.05,0.1,0.3$, and $1.5 \mathrm{~L}$, respectively. In these plots, the solid lines indicate the solution for the new model and the dashed lines indicate the solution for the Reduced Model. For the chosen parameters, oscillations in ventilation occur for the Reduced Model for very small compartment volumes, as shown in Fig. $4 \mathrm{a}$ when $V_{\mathrm{A}_{1}}=0.05 \mathrm{~L}$. However, these oscillations cease when the volume is increased to $V_{\mathrm{A}_{1}}=0.3 \mathrm{~L}$, as shown in Fig. 4c. This is in contrast to the new model that allows for alveolar collapse, which does exhibit oscillations at the higher resting volume, indeed up to a volume of $1.5 \mathrm{~L}$ as shown in Fig. 4d, although the oscillations are very small.

These plots demonstrate that the amplitude of oscillation has increased for the new model-now $\dot{V}_{\mathrm{I}}$ oscillates between approximately 4 and $13 \mathrm{~L} \mathrm{~min}^{-1}$, whereas previously the range was between 5.2 and $9.9 \mathrm{~L} \mathrm{~min}^{-1}$. This highlights that the new model displays periods of normal breathing where the inspired ventilation is equal to $\dot{V}_{0}$ at its lowest point. This occurs when the arterial oxygen partial pressure exceeds $104 \mathrm{mmHg}$, as defined by Eq. (2). In contrast, the Reduced Model does not attain this value. The maximum ventilation is limited by the minimum arterial oxygen partial pressure that is obtained. Further to this, the period of oscillation has increased to approximately $60 \mathrm{~s}$. Both of these observations are in closer agreement with Cheyne-Stokes breathing. ${ }^{14}$
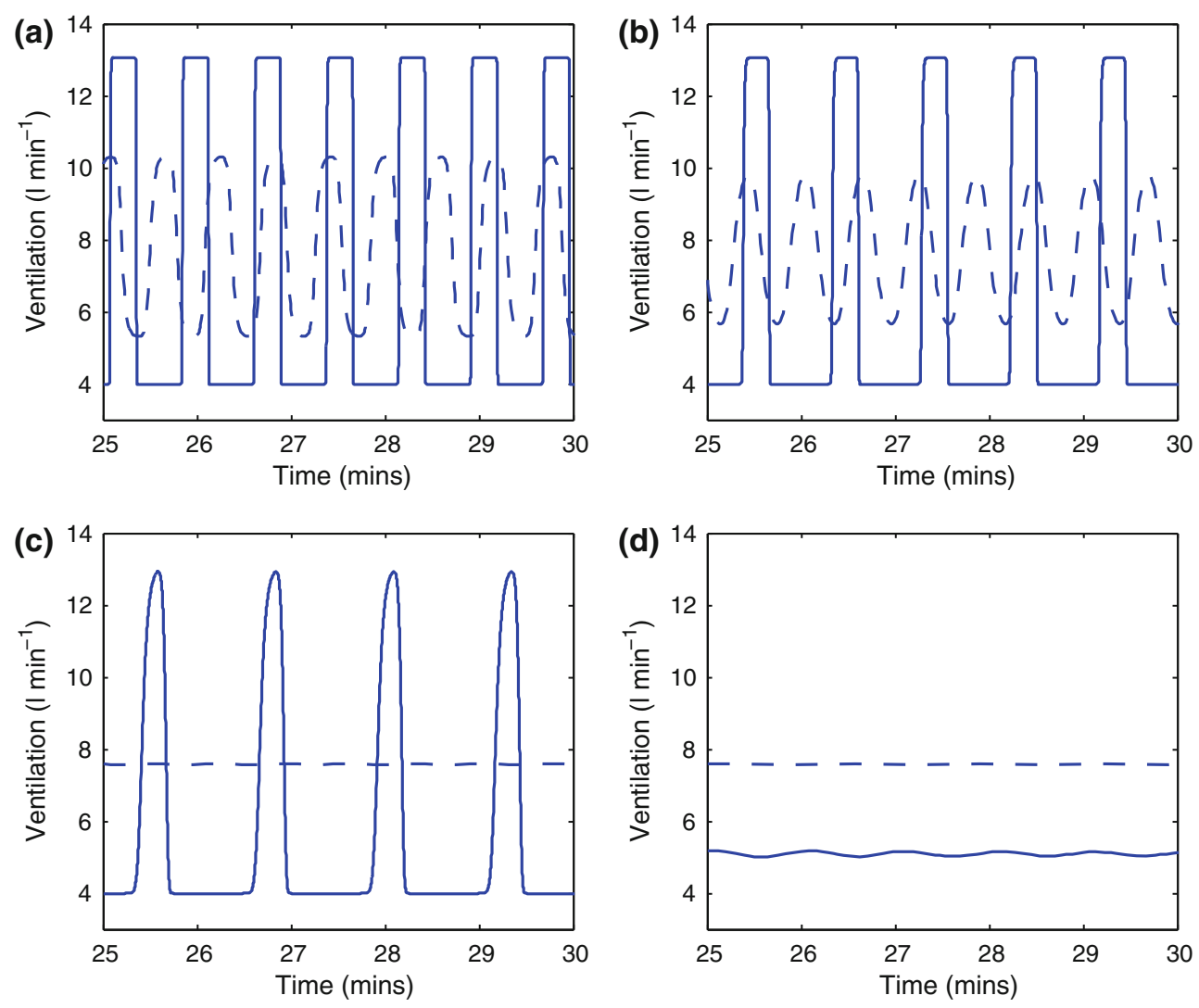

FIGURE 4. Comparison of the oscillations in the total inspired ventilation for the Reduced Model (dashed line), independently reproduced and in agreement with the original results, ${ }^{24}$ with the model that allows variable compartment volume (solid line). (a) $V_{\mathrm{A}_{1}}=V_{\mathrm{A}_{1} \text { rest }}=0.05 \mathrm{~L}$; (b) $V_{\mathrm{A}_{1}}=V_{\mathrm{A}_{1} \text { rest }}=0.1 \mathrm{~L}$; (c) $V_{\mathrm{A}_{1}}=V_{\mathrm{A}_{1} \text { rest }}=0.3 \mathrm{~L}$; (d) $V_{\mathrm{A}_{1}}=V_{\mathrm{A}_{1} \text { rest }}=1.5 \mathrm{~L}$. 
In particular though, it is clear that the shape of the oscillations differs between the two models. As outlined earlier, the Reduced Model predicts smooth, regular oscillations. The results using the new model, however, show short periods of normal breathing, where $\dot{V}_{\mathrm{I}}=\dot{V}_{0}$, punctuated by intervals of high ventilation; behavior characteristic of Cheyne-Stokes breathing. Thus, the results of the extended model are more consistent with experimentally observed behavior of subjects suffering from periodic breathing, and hence demonstrate an improvement over existing results.

\section{Effect of $V_{\mathrm{A}_{1}}$}

The results of the Reduced Model indicate that periodic breathing only occurs in small, poorly ventilated lung compartments, and the volume of the compartment is defined to remain constant. A resting volume of $0.3 \mathrm{~L}$ is assumed in the results of the extended model, shown in Figs. 3 and 4. Figure 5 compares the results when varying the rest volume $V_{\mathrm{A}_{1} \text { rest }}=0.05$ (dot-dashed line), 0.1 (dotted line), 0.3 (solid line), and 1.5 L (dashed line), showing the effect on the oscillations in compartment volume in the new model. Figure 5 reveals that oscillations in compartment volume are observed for the lower resting volumes, but cease for a very large resting volume, $V_{\mathrm{A}_{1} \text { rest }}=1.5 \mathrm{~L}$. This is in agreement with Fig. 4, which shows that the oscillations in $\dot{V}_{\mathrm{I}}$ cease for $V_{\mathrm{A}_{1} \text { rest }}=$ $1.5 \mathrm{~L}$. Previous studies also agree with these results, as findings show that respiratory stability is increased with larger volume, such that the lung volume acts to damp oscillations. ${ }^{15,22}$ A feature of the model demonstrated by these plots is that periodic oscillations can be observed at larger compartment volumes than in the Reduced Model, which is a more physiologically realistic result.

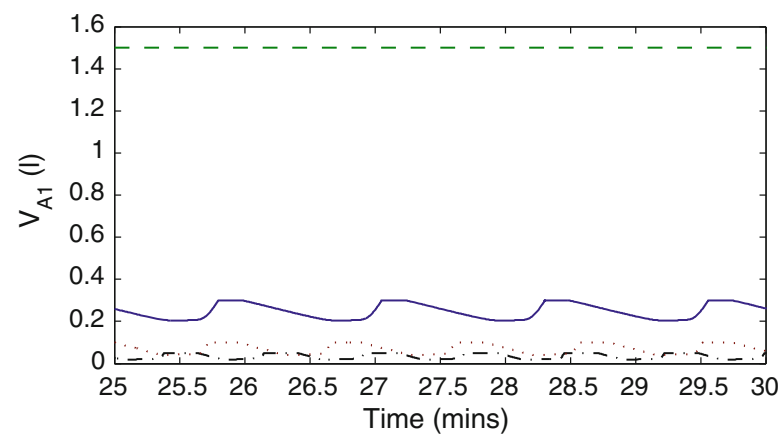

FIGURE 5. Response of the model that allows alveolar collapse to changes in the resting volume of the poorly ventilated compartment, $V_{\mathrm{A}_{1} \text { rest }}: V_{\mathrm{A}_{1} \text { rest }}=0.05$ (dot-dashed line), $V_{\mathrm{A}_{1} \text { rest }}=$ 0.1 (dotted line), 0.3 (solid line), and $1.5 \mathrm{~L}$ (dashed line). This plot shows oscillations in $V_{\mathrm{A}_{1}}$ as a function of time, with $\delta_{\mathrm{s}}=0.055 \mathrm{~L}, F_{1}=0.8, v_{1}=0.12, P_{\mathrm{BCO}_{2}}=41 \mathrm{mmHg}$.

\section{Effect of $F_{I}$}

It is known that inspired oxygen concentration affects the development of atelectasis, and increasing the fraction of inspired oxygen has been found to reduce the time taken to collapse. ${ }^{3,11,12}$ Given that lung collapse is known to occur when a subject is administered high levels of oxygen, this parameter is assigned a value of 0.8 for the extended model. Figure 6 compares the results for $F_{\mathrm{I}}=0.8$ (solid line) with the effects of a normal inspired oxygen level, $F_{\mathrm{I}}=0.21$ (dotted line), and the equivalent of a patient being administered $100 \%$ oxygen, $F_{\mathrm{I}}=1$ (dashed line). In agreement with experimental observation, collapse of the poorly ventilated compartment does not occur for $F_{\mathrm{I}}=0.21$, but the volume remains constant at the rest value, shown in Fig. 6a. The amplitude of oscillations, and thus the extent of collapse, increases with increasing level of inspired oxygen.

As the level of inspired oxygen increases, so does the maximum fractional concentration of oxygen in the compartment, as demonstrated in Fig. 6b. This in turns causes an increase in the maximum arterial oxygen partial pressure and content. ${ }^{24}$ Figure $6 \mathrm{c}$ is in agreement with this result, and furthermore shows that the amplitude of oscillations in $P_{\mathrm{aO}_{2}}$ also increases with $F_{\mathrm{I}}$. Lastly, Fig. $6 \mathrm{~d}$ shows that the amplitude of oscillations in ventilation increases as the level of inspired oxygen decreases. This result occurs as a subject will need to breathe more deeply if there is less oxygen in the inspired air.

\section{Effect of $\delta_{s}$}

$\delta_{\mathrm{s}}$ is the correction term that accounts for the inspired oxygen that remains in the alveolar deadspace of the poorly ventilated compartment, and therefore does not contribute to the actual amount of gas entering the region. The results given in the section "Summary of Computations" take $\delta_{\mathrm{s}}=0.055 \mathrm{~L}$, and Fig. 7 shows additional results for $\delta_{\mathrm{s}}=0.04$ and $0.09 \mathrm{~L}$.

As $\delta_{\mathrm{s}}$ increases, Eqs. (5) and (6) show that the amount of inspired, and consequently the amount of expired, oxygen to the compartment decreases. $\dot{V}_{\mathrm{E}_{1}}$ becomes negative more rapidly, and thus the volume of the compartment will decrease at a greater rate. This is observed in Fig. 7a, where the oscillations in $V_{\mathrm{A}_{1}}$ increase in amplitude as $\delta_{\mathrm{s}}$ increases, so that the extent of compartment collapse increases. For small $\delta_{\mathrm{s}}$, the volume of the compartment remains at the rest volume, at which point $\dot{V}_{\mathrm{E}_{1}}$ is positive. Figures $7 \mathrm{~b}$ and $7 \mathrm{c}$ show that the oscillations in $F_{\mathrm{A}_{1}}$ and $P_{\mathrm{aO}_{2}}$ increase as $\delta_{\mathrm{s}}$ increases, respectively. This follows as a larger alveolar deadspace will lower the amount of oxygen entering the poorly ventilated compartment. In contrast to the 

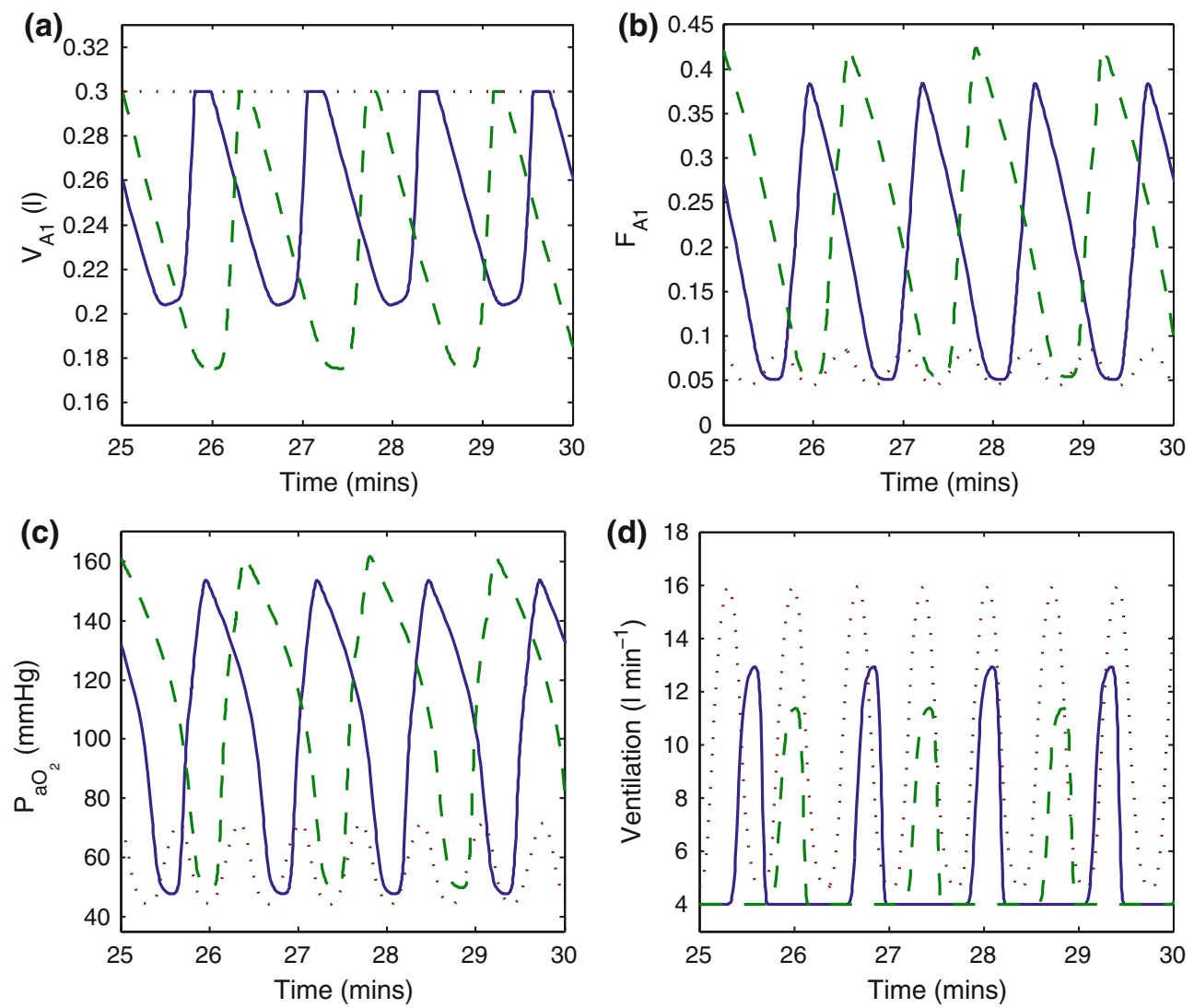

FIGURE 6. Response of the model that allows alveolar collapse to changes in the level of inspired oxygen, $F_{1}: F_{1}=0.21$ (dotted line), 0.8 (solid line), 1 (dashed line) (a) $V_{\mathrm{A}_{1}}$; (b) $F_{\mathrm{A}_{1}}$; (c) $P_{\mathrm{aO}_{2}}$; (d) $V_{1}$, all shown as a function of time. Here, $\delta_{\mathrm{s}}=0.055 \mathrm{~L}, V_{\mathrm{A}_{1} \text { rest }}=0.3 \mathrm{~L}$, $v_{1}=0.12, P_{\mathrm{BCO}_{2}}=41 \mathrm{mmHg}$.

results shown in the section "Effect of $F_{\mathrm{I}}$," however, the total inspired ventilation decreases accordingly, which reveals a subtle sensitivity in the model to the alveolar deadspace. In this situation, a larger alveolar deadspace results in a more unhealthy subject, who would have to increase overall ventilation, $\dot{V}_{\mathrm{I}}$, in order to deliver adequate oxygen to the compartment, $\dot{V}_{\mathrm{I}_{1}}$.

\section{DISCUSSION}

This article presents an extension to the Reduced Model of periodic breathing proposed by Whiteley et $a .^{24}$ to take into account non-constant compartment volume, thereby investigating incidence of pulmonary collapse in a poorly ventilated compartment of the lung. In consideration of the conditions under which periodic breathing can arise, and as poorly ventilated lung compartments are vulnerable to collapse, this study is motivated by the hypothesis that a more comprehensive link can be demonstrated between atelectasis and periodic breathing.

The results of this new work predict regular oscillations in lung volume, which coincide with oscillations in alveolar oxygen concentration, arterial partial pressure, and inspired ventilation. The degree of collapse is found to be dependent on the fraction of inspired oxygen and ventilation supplied to the compartment, in agreement with experimental observations. It has also been shown that by increasing the term $\delta_{\mathrm{s}}$ that quantifies the alveolar deadspace-a consequence of the existence of a poorly ventilated compartment - the extent of compartment collapse increases.

Furthermore, two of the weaknesses of the Reduced Model have been addressed by this work: (i) the shape of the oscillations in ventilation is more accurate, and in closer agreement with experimentally observed behavior of the nature of periodic breathing; and (ii) the observed oscillations occur at higher compartment volumes than found previously. Thus, the new model has removed the unrealistic restrictions placed on compartment volume that were required by the Reduced Model for periodic breathing to occur. Indeed an analysis of the response of the model to the different parameters shows that oscillations will occur for resting volumes up to around $1.5 \mathrm{~L}$, and that decreasing the amplitude of oscillations in $V_{\mathrm{A}_{1}}$ coincides with a 

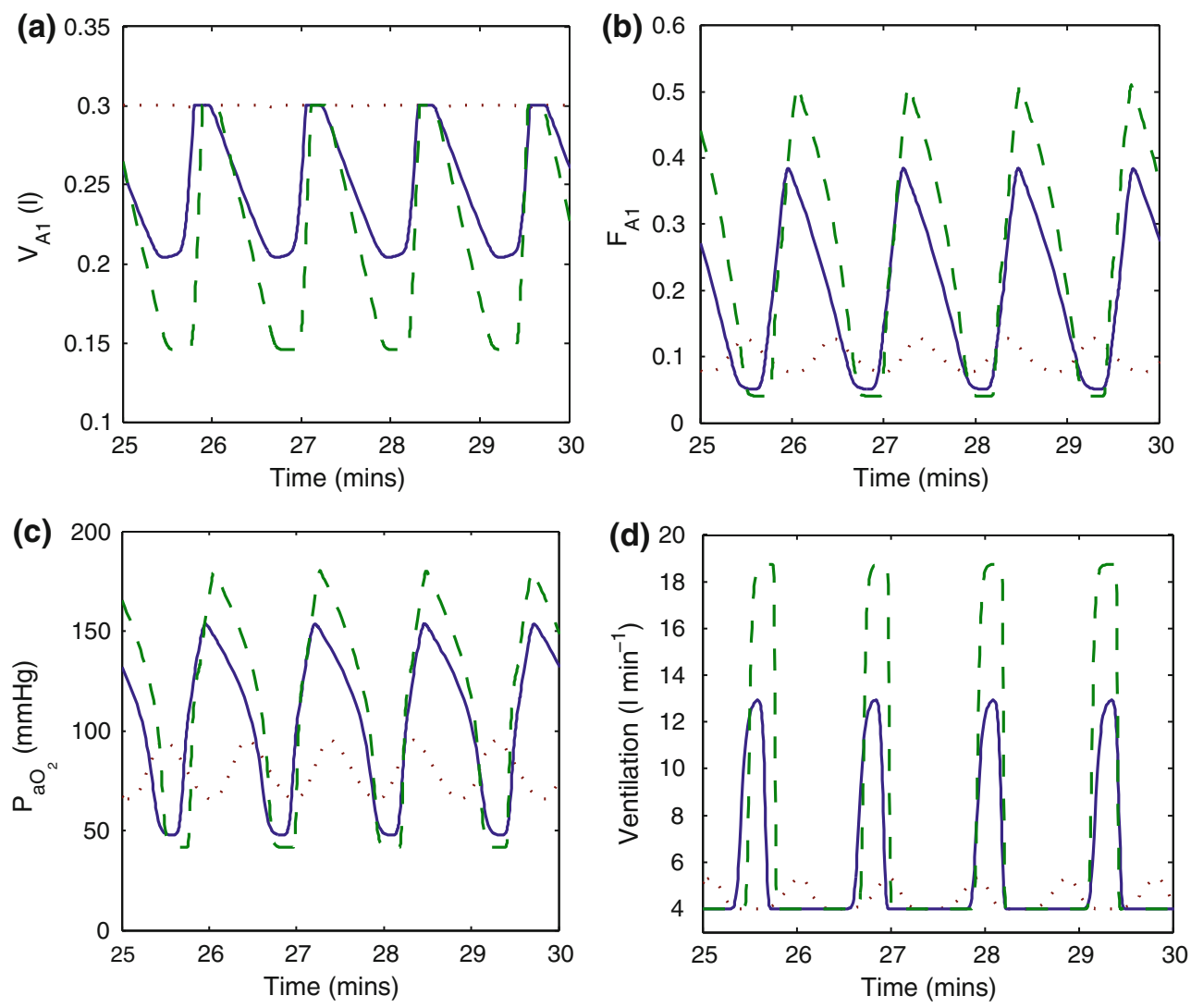

FIGURE 7. Response of the model that allows alveolar collapse to changes in the amount of inspired gas that remains in the dead space, $\delta_{\mathrm{s}}: \delta_{\mathrm{s}}=0.04$ (dotted line), 0.055 (solid line), and $0.09 \mathrm{~L}$ (dashed line) (a) $V_{\mathrm{A}_{1}} ;$ (b) $F_{\mathrm{A}_{1}} ;$ (c) $P_{\mathrm{aO}_{2}} ;$ (d) $V_{1}$. Here, $F_{1}=0.8$, $V_{\mathrm{A}_{1} \text { rest }}=0.3 \mathrm{~L}, V_{1}=0.12, P_{\mathrm{BCO}_{2}}=41 \mathrm{mmHg}$.

decrease in those in $\dot{V}_{\mathrm{I}}$. This reveals the correlation between periodic breathing and compartment volume. Hence, the model for periodic breathing that allows for alveolar collapse accurately predicts the expected oscillations, and with increasing accuracy over existing models. This in turn establishes the requirement for variable compartment volume to accurately investigate periodic breathing.

It is still the case, however, that there are limitations inherent in this model, which can be addressed in future iterations. Firstly, it is assumed that the poorly ventilated lung compartment behaves independently of the healthy second compartment. As such, it cannot benefit from gas contribution by the healthy compartment, should the lung act to maintain homogeneous gas pressure throughout under adverse conditions. This assumption is not unrealistic if one considers a completely blocked airway, as in such a case it is easier for gas to travel to the healthy compartment but not from the healthy compartment to the poorly ventilated compartment. In the case of a partially blocked airway, however, it is plausible that the lung will be able to maintain gas pressures throughout, and draw gas from the healthy compartment if necessary. This must be considered in order to take this model forward. Nevertheless, if poor ventilation to a region of the lung is caused by a restriction to gas flow in the airways, it is likely that a poorly ventilated compartment will not benefit fully from gas available elsewhere in the lungs.

Secondly, while these results yield further insight into the effect of lung volume on periodic breathing, and reveal more realistic oscillations in ventilation than those previously observed, the model does not predict periods of apnoea, which are commonly present in patients exhibiting Cheyne-Stokes breathing. This is due to the assumption of a base level of ventilation, which is attained periodically throughout the oscillations. A more reliable response is required to predict little or no ventilation and this is therefore a direction for further research. Also, as an average-flow model, it is assumed that there is a continuous flow of gas to and from the poorly ventilated compartment. Consequently, $\dot{V}_{\mathrm{I}_{1}}$ and $\dot{V}_{\mathrm{E}_{1}}$ are average ventilation expressions defined at all time points, rather than unique inspiration and expiration phases of tidal volume. This is a further limitation of the model that can be addressed as an extension to this study. 


\section{APPENDIX A: THE SOLUTION METHOD}

In this section, the relationships between $F, P, C$ are defined, as well as those between $G$ and $D$ for the carbon dioxide. Parameter values are given in Table 3.

The alveolar partial pressure is found by applying Dalton's law, which states that the partial pressure of a gas in a gas mixture is the pressure that this gas would exert if it occupied the total volume ${ }^{17}$ :

$$
P_{\mathrm{A}_{1}}=\left(P_{\mathrm{B}}-P_{\mathrm{H}_{2} \mathrm{O}}\right) F_{\mathrm{A}_{1}} .
$$

Here, $P_{\mathrm{B}}$ is the atmospheric pressure and $P_{\mathrm{H}_{2} \mathrm{O}}$ the partial pressure of water vapor. Since compartment 2 is defined to be the healthy region, the $\mathrm{O}_{2}$ blood content in it is assumed constant.

Alveolar oxygen partial pressure is then used to find the oxygen content in the blood leaving compartment 1 via

$$
C_{\mathrm{A}_{1}}=K_{\mathrm{Hb}} S\left(P_{\mathrm{A}_{1}}\right)+\alpha P_{\mathrm{A}_{1}} .
$$

The constants $K_{\mathrm{Hb}}$ and $\alpha$ represent the oxygen carrying capacity of hemoglobin and the solubility coefficient of oxygen in the blood, respectively. $S(P)$ is the saturation function, defined empirically by

$$
S(P)=\frac{a_{1} P+a_{2} P^{2}+a_{3} P^{3}+P^{4}}{a_{4}+a_{5} P+a_{6} P^{2}+a_{7} P^{3}+P^{4}},
$$

which gives the fractional oxygen saturation of the blood.

Equation (A.2) is used to find $C_{\mathrm{A}_{2}}$, which will be assumed constant. Thus, having found $C_{\mathrm{A}_{1}}$ and $C_{\mathrm{A}_{2}}$, the total alveolar oxygen content can be determined as the weighted sum of its components from each region, according to the fraction of total perfusion that they receive:

$$
C_{\mathrm{A}}=\frac{Q_{1}}{Q_{1}+Q_{2}} C_{\mathrm{A}_{1}}(t)+\frac{Q_{2}}{Q_{1}+Q_{2}} C_{\mathrm{A}_{2}} .
$$

Under the assumption that diffusion across the alveolar membrane is instantaneous, arterial oxygen content is equilibrated with alveolar content, i.e., $C_{\mathrm{a}}=C_{\mathrm{A}}$. Then the total arterial partial pressure can be found by solving (A.2) for $P_{\mathrm{a}}$ :

$$
C_{\mathrm{a}}(t)-K_{\mathrm{Hb}} S\left(P_{\mathrm{a}}(t)\right)-\alpha P_{\mathrm{a}}(t)=0 .
$$

The carbon dioxide content in the mixed venous blood, $D_{\bar{v}}$, and in the poorly ventilated compartment, $D_{\mathrm{A}_{1}}$, are calculated using the following equations.

$$
\begin{aligned}
K D_{\bar{v}} & =\lambda\left(G_{\bar{v}}+G_{0}\right), \\
K D_{\mathrm{A}_{1}} & =\lambda\left(G_{\mathrm{A}_{1}}+G_{0}\right) .
\end{aligned}
$$

The constant $\lambda$ is the incremental $\mathrm{CO}_{2}$ solubility in blood, and $G_{0}$ a base value for the fractional concentration. $G_{\bar{v}}$ is calculated by assuming a value of $P_{\bar{\nu} \mathrm{CO}_{2}}=46 \mathrm{mmHg}$ and applying (A.1).

The arterial oxygen partial pressure, $P_{\mathrm{a}}$, is used to calculate the total inspired ventilation according to Eq. (2), and from this $\dot{V}_{I_{1}}$ is found using (5). $\dot{V}_{\mathrm{I}_{1}}$ is used to calculate the amount of expired ventilation, $\dot{V}_{\mathrm{E}_{1}}$ from (6). The differential equation (7) is solved for $V_{\mathrm{A}_{1}}$, and then those for $F_{\mathrm{A}_{1}}$ and $G_{\mathrm{A}_{1}}$ using the Matlab delay differential equation solver, dde23.

\section{ACKNOWLEDGMENT}

Sara-Jane Dunn is supported by an EPSRC-funded Life Sciences Interface Doctoral Training Centre studentship (Grant No. EP/E501605/1).

\section{REFERENCES}

${ }^{1}$ Batzel, J. J., and H. T. Tran. Stability of the human respiratory control system. J. Math. Biol. 41:45-79, 2000.

${ }^{2}$ Carley, D. W., and D. C. Shannon. A minimal mathematical model of human periodic breathing. J. Appl. Physiol. 65:1400-1409, 1988.

${ }^{3}$ Duggan, M., and B. P. Kavanagh. Pulmonary atelectasis, a pathogenic perioperative entity. Anesthesiology 102:838854, 2005.

${ }^{4}$ El Hefnawy, A., G. M. Saidel, and E. N. Bruce. $\mathrm{CO}_{2}$ control of the respiratory system: plant dynamics and stability analysis. Ann. Biomed. Eng. 16:445-461, 1988.

${ }^{5}$ Fletcher, R., B. Jonson, G. Cumming, and J. Brew. The concept of deadspace with special reference to the single breath test for carbon dioxide. Br. J. Anaesth. 53:77-88, 1981.

${ }^{6}$ Fowler, A. C. Mathematical Models in the Applied Sciences. Cambridge: Cambridge University Press, 1997.

${ }^{7}$ Fowler, A. C., and G. P. Kalamangalam. The role of the central chemoreceptor in causing periodic breathing. IMA J. Math. Appl. Med. Biol. 17:147-167, 2000.

${ }^{8}$ Fowler, A. C., G. P. Kalamangalam, and G. Kember. A mathematical analysis of the Grodins model of respiratory control. IMA J. Math. Appl. Med. Biol. 10:249-280, 1993.

${ }^{9}$ Franklin, K. A., E. Sandstrom, G. Johansson, and E. M. Balfors. Hemodynamics, cerebral circulation, and oxygen saturation in Cheyne-Stokes respiration. J. Appl. Physiol. 83:1184-1191, 1997.

${ }^{10}$ Grodins, F. S., J. Buell, and A. J. Bart. Mathematical analysis and digital simulation of the respiratory control system. J. Appl. Physiol. 22:260-276, 1967.

${ }^{11}$ Joyce, C. J., A. B. Baker, and R. R. Kennedy. Gas uptake from an unventilated area of lung: computer model of absorption atelectasis. J. Appl. Physiol. 74:1107-1116, 1993.

${ }^{12}$ Joyce, C. J., and A. B. Williams. Kinetics of absorption atelectasis during anesthesia: a mathematical model. J. Appl. Physiol. 86:1116-1125, 1999.

${ }^{13}$ Kavanagh, B. P. Perioperative atelectasis. Minerva Anestesiol. 74:285-287, 2008.

${ }^{14}$ Keener, J., and J. Sneyd. Mathematical Physiology. New York: Springer, 1998. 
${ }^{15}$ Khoo, M. C. K., R. E. Kronauer, K. P. Strohl, and A. S. Slutsky. Factors inducing periodic breathing in humans: a general model. J. Appl. Physiol. 53:644-659, 1982.

${ }^{16}$ Longobardo, G. S., N. S. Cherniack, and B. Gothe. Factors affecting respiratory system stability. Ann. Biomed. Eng. 17:377-396, 1989.

${ }^{17}$ Lumb, A. B. Nunn's Applied Respiratory Physiology, 5th ed. Oxford: Butterworth Heinemann, 2000.

${ }^{18}$ Mackey, M. C., and L. Glass. Oscillation and chaos in physiological control systems. Science 197:287-289, 1977.

${ }^{19}$ Pinna, G. D., R. Maestri, A. Mortara, M. T. La Rovere, F. Fanfulla, and P. Sleight. Periodic breathing in heart failure patients: testing the hypothesis of instability of the chemoreflex loop. J. Appl. Physiol. 89:2147-2157, 2000.

${ }^{20}$ Reeder, M. K., M. D. Goldman, L. Loh, A. D. Muir, and K. R. Casey. Haemodynamic effects of periodic ventilation abolition with supplementary oxygen. Br. J. Anaesth. 67:326-328, 1991.
${ }^{21}$ Severinghaus, J. W., and M. Stupfel. Alveolar dead space as an index of distribution of blood flow in pulmonary capillaries. J. Appl. Physiol. 10:335-348, 1957.

${ }^{22}$ Vielle, B., and G. Chauvet. Delay equation analysis of human respiratory stability. Math. Biosci. 152:105-122, 1998. doi:10.1016/S0025-5564(98)10028-7.

${ }^{23}$ Whiteley, J. P., D. J. Gavaghan, and C. E. W. Hahn. Mathematical modelling of pulmonary gas transport. J. Math. Biol. 47:79-99, 2003.

${ }^{24}$ Whiteley, J. P., D. J. Gavaghan, and C. E. W. Hahn. Periodic breathing induced by arterial oxygen partial pressure oscillations. Math. Med. Biol. 20:205-224, 2003.

${ }^{25}$ Whiteley, J. P., M. J. Turner, A. B. Baker, D. J. Gavaghan, and C. E. W. Hahn. The effects of ventilation pattern on carbon dioxide transfer in three computer models of the airways. Respir. Physiol. Neurobiol. 131:269-284 2002. doi: 10.1016/S1569-9048(02)00066-6. 